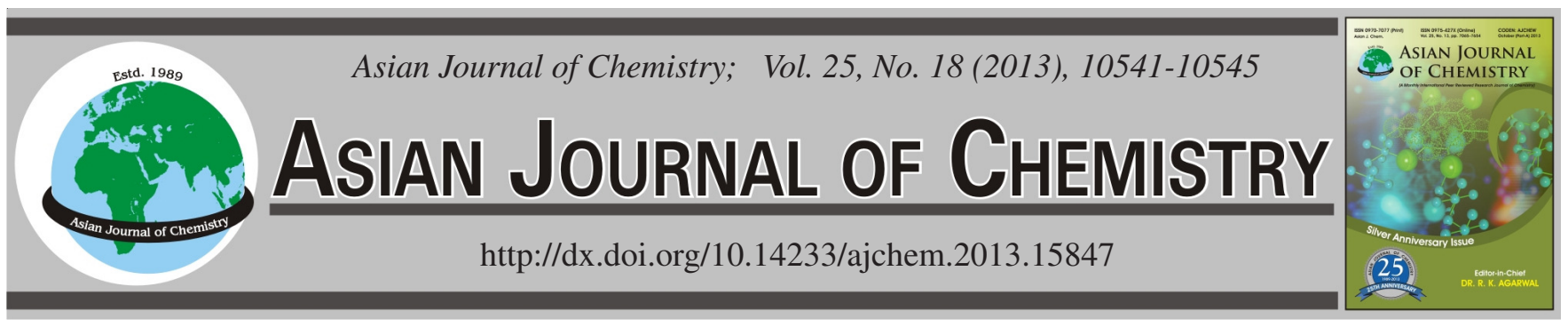

\title{
Label Free Detection of Adenosine Based on Hemin/G-Quadruplex Peroxidase-Mimicking DNAzyme as Bioelectrocatalyst
}

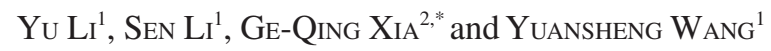

\begin{abstract}
${ }^{1}$ Department of Chemistry and Materials, College of Science, The Naval University of Engineering, Wuhan 430022, Hubei, P.R. China ${ }^{2}$ Department of Obstetrics and Gynecology, Union Hospital, Tongji Medical College, Huazhong University of Science and Technology,
\end{abstract} Wuhan 430022, Hubei, P.R. China

*Corresponding author: Fax: +86 27 83443066; Tel: +86 27 68771774, E-mail: chancellery11@gmail.com

(Received: 30 May 2013;

Accepted: 26 November 2013)

AJC-14443

\begin{abstract}
The hemin/G-quadruplex horseradish peroxidase-mimicking DNAzyme is assembled on gold electrodes. It reveals bioelectrocatalytic properties and electrocatalyzes the reduction of $\mathrm{H}_{2} \mathrm{O}_{2}$. The bioelectrocatalytic functions of the hemin/G-quadruplex DNAzyme is used to develop electrochemical sensor for the detection of adenosine. Hairpin nucleic structure that includes the G-quadruplex sequence in a caged configuration and the nucleic acid sequence complementary to the aptamer sequence for adenosine, is immobilized on goldelectrode surfaces. In the presence of the analyte, the hairpin structure is opened and the hemin/G-quadruplex horseradish peroxidasemimicking DNAzyme structure is generated on the electrode surfaces. The bioelectrocatalytic cathodic current generated by the functionalized electrode, upon the electrochemical reduction of $\mathrm{H}_{2} \mathrm{O}_{2}$, provides a way for the detection of adenosine.
\end{abstract}

Key Words: Adenosine, Label free, DNAzyme, Electrochemical detection.

\section{INTRODUCTION}

Standard DNA and RNA aptamers themselves are not inherently catalyst, most of aptamer sensors require an additional modified process. Some studies have implemented metal nanoparticles in the development of optical ${ }^{1}$, electrical ${ }^{2}$, fieldeffect transistor ${ }^{3}$, or microgravimetric nucleic acids-based biosensors ${ }^{4}$, the others are toward the development of enzymes ${ }^{5}$ or metal nanoparticles ${ }^{6}$ as catalytic labels for amplifying DNAsensing or aptamer-sensing events. In these systems, the DNAzymes (catalytic nucleic acids) play, however, a passive role whereby the electroactive label or the electroluminescencegenerating labels are cleaved off from the DNAzyme-substrate complexes to yield readable signals ${ }^{7}$. These systems lack, however, a bioelectrocatalytic amplification path. In fact, the direct use of DNAzymes as electrocatalytic labels for amplifying sensing events is unexplored.

One of the most explored DNAzymes for bioanalytical applications is the horseradish-peroxidase-mimicking DNAzyme that consists of hemin, which is stacked in a Gquadruplex nucleic acid structure $^{8}$. Different colorimetric ${ }^{9}$ or chemiluminescent ${ }^{10}$ sensing schemes for analyzing DNA, aptamer-substrate complexes, or metal ions using the hemin/ G-quadruplex horseradish-peroxidase-mimicking DNAzyme have been developed and nucleic acid based "machines" that generate the hemin/G-quadruplex DNAzyme upon the sensing of DNA ${ }^{11}$, proteins ${ }^{12}$ or metal ions ${ }^{13}$ have been investigated for the ultrasensitive detection of the different analytes. Besides the catalytic activities of the hemin/G-quadruplex DNAzyme, the hemin/G-quadruplex was also found to act as an electrocatalyst for the reduction of $\mathrm{H}_{2} \mathrm{O}_{2}$ and this was used to develop amplified electrochemical DNA sensors or aptasensors ${ }^{14}$.

Among the signal transduce techniques adopted on these aptasensors, electrochemical methodology is considered to have technical simplicity, good sensitivity and easy adaptability for in situ analysis with relatively low cost instrumental set-ups ${ }^{15}$. In this study, we report on the construction of a labelfree and sensitive electrochemical aptasensor for adenosine by incorporating a peroxidase-mimicking DNAzyme sequence into the hairpin aptamer probe. The presence of adenosine opens the hairpin structure of the aptamer probe and forms a hemin/G-quadruplex with the aid of hemin, which exhibits a biocatalytic capability towards electro-reduction of $\mathrm{H}_{2} \mathrm{O}_{2}$ and generates an amplified electrochemical signal for adenosine monitoring.

\section{EXPERIMENTAL}

4-(2-Hydroxyethyl)-1-piperazine ethanesulfonic acid (HEPES), dimethyl sulfoxide (DMSO),6-mercapto-1-hexanol $(\mathrm{MCH})$, adenosine and hemin were purchased from Sigma (Shanghai, China), uridine, guanosine, cytidine and the hair- 
pin aptamer were received from Shanghai Sangon Biological Engineering Technology and Services Co., Ltd. (Shanghai, China). The aptamer construct was designed using known sequence for the peroxidase-mimicking DNAzyme. The correct folding and thermodynamic parameters $\left(\Delta \mathrm{G}^{\circ}\right.$ and melting temperature) were evaluated using the Oligo-Analyzer program (at http://mfold.rna.albany.edu/), The aptamer was HPLC-purified and freeze-dried by the supplier and the sequence is as follows: 5'- ACCTGGG GGAGT ATTGC GGAGGAAGGT GGGTA GGGCG GGTTG GG CCCCAGGT$\left(\mathrm{CH}_{2}\right) 6-\mathrm{SH} 3$ ', the sequence underlined is the DNAzyme (A), while the bold one is aptamer for adenosine (B). The stock solution of hemin was prepared in dimethyl sulfoxide and stored in the dark at $20{ }^{\circ} \mathrm{C}$ for later experiments. Ultrapure water was used throughout the experiments.

Modification of the electrodes: The gold electrode (diameter $=3 \mathrm{~mm}$ ) was treated according to a documented procedure $^{16}$, which was first cleaned with a "piranha"solution (mixture of concentrated $\mathrm{H}_{2} \mathrm{SO}_{4}$ and $\mathrm{H}_{2} \mathrm{O}_{2}$ at volume ratio of $3: 1)$, polished carefully with alumina $(0.3 \mathrm{~mm}$ and $0.05 \mathrm{~mm}$ in diameter) suspensions and washed with water. Then the electrode was electrochemically cleaned in $0.5 \mathrm{M}$ sulfuric acid until a stable cyclic voltammogram was obtained. Finally, the electrode was washed with water and ethanol, respectively and dried with nitrogen.

The aptamer, diluted with $10 \mathrm{mM}$ HEPES buffer (100 $\mathrm{mM} \mathrm{NaCl}, 50 \mathrm{mM} \mathrm{KCl}, \mathrm{pH}$ 7.2), was incubated at room temperature with the gold electrode for overnight (more than $8 \mathrm{~h}$ ), followed by surface blocking with $\mathrm{MCH}(2 \mathrm{mM})$ for $1 \mathrm{~h}$. Then the electrode was washed with $10 \mathrm{mM}$ HEPES buffer. Subsequently, the electrode was incubated with different concentrations of adenosine for $60 \mathrm{~min}$. After washing with $10 \mathrm{mM}$ HEPES buffer, hemin $(2 \mu \mathrm{M})$ was incubated with the electrode for $30 \mathrm{~min}$ to form a stable hemin/G-quadruplex peroxidasemimicking DNAzyme structure.

Electrochemical detection of adenosine: The resulting electrode was placed in a glass cell containing $50 \mathrm{~mL} 10 \mathrm{mM}$ HEPES buffer ( $100 \mathrm{mM} \mathrm{NaCl}, 50 \mathrm{mM} \mathrm{KCl}, \mathrm{pH} 7.2)$ and $1 \mathrm{mM}$ $\mathrm{H}_{2} \mathrm{O}_{2}$ and connected to an electrochemical workstation (CHI 660 C, CH Instruments Inc., Shanghai, China) for measurements. A standard three electrodes configuration with the modified electrode as the working electrode, a platinum wire as the counter electrode and a saturated calomel electrode (SCE) as the reference electrode was used for the measurements. Before the measurements, the HEPES buffer solution is bubbled thoroughly with nitrogen for $10 \mathrm{~min}$. Cyclic voltammograms $(\mathrm{CVs})$ were recorded by cycling the potential between -0.8 to $0 \mathrm{~V}$ at a scan rate of $10 \mathrm{mVS}^{-1}$ and the current responses at -0.8 $\mathrm{V}$ were chosen as the analytical signals.

\section{RESULTS AND DISCUSSION}

Scheme: The hemin/G-quadruplex HRP-mimicking DNAzyme is extensively used as a biocatalyst for an amplified biosensor through the catalyzed oxidation of 2,2'-azinobis(3ethyl-benzthiazoline-6-sulfonic acid) (ABTS-) to coloured $\mathrm{ABTS}^{17,18}$. whereas there were only a few research projects on the electrocatalytic activity of hemin/G-quadruplex DNAzyme ${ }^{16,19}$. The signal was observed not from the DNAzyme but from the substrate catalyzed by the DNAzyme during the electrochemical procedure. In a pioneer work, Willner et al. ${ }^{18,20-22}$ discussed the potential electrocatalytic applications of hemin/Gquadruplex DNAzyme. For the preparation of peroxidasemimicking DNAzyme-based electrochemical biosensors, the DNA strand, which can form G-quadruplex, was first immobilized on the electrode surface. After being incubated with hemin, DNAzyme was produced, which catalyzed the reduction of $\mathrm{H}_{2} \mathrm{O}_{2}$ electrochemically.

Here, we construct an electrochemical aptasensor for adenosine based on the peroxidase-mimicking DNAzyme as the electrochemical amplification label. For this purpose, we design a hairpin aptamer probe which consists of two kinds of nucleic acid sequences ( $\mathrm{A}$ and $\mathrm{B}$, as described earlier), the peroxidase-mimicking DNAzyme sequence $\mathrm{A}$ and adenosinebinding sequence $\mathrm{B}$. The sequence $\mathrm{A}$ can interact with hemin to form a hemin/G-quadruplex DNAzyme structure and catalyze the reduction of $\mathrm{H}_{2} \mathrm{O}_{2}$. The principle of this aptasensor is illustrated in Scheme-I.

The hairpin aptamer is first self-assembled on a gold electrode surface through $\mathrm{Au}-\mathrm{S}$ bonds and the surface is then blocked with $\mathrm{MCH}$ to reduce non-specific adsorption. In the absence of adenosine, the hairpin aptamer is closed and there is no interaction between sequence B and hemin. In this case, no obvious current response is observed. However, in the presence of the target adenosine, the hairpin aptamer opens and releases sequence $\mathrm{B}$, which combines with hemin to form a hemin/G-quadruplex peroxidase mimicking DNAzyme structure to electrocatalyze the reduction of $\mathrm{H}_{2} \mathrm{O}_{2}$ and generates the cathodic current for measurement. At the mean time, the degree of opening of the hairpin aptamer is related to the concentration of adenosine. An elevated concentration of adenosine leads to increased binding of hemin to sequence $\mathrm{B}$

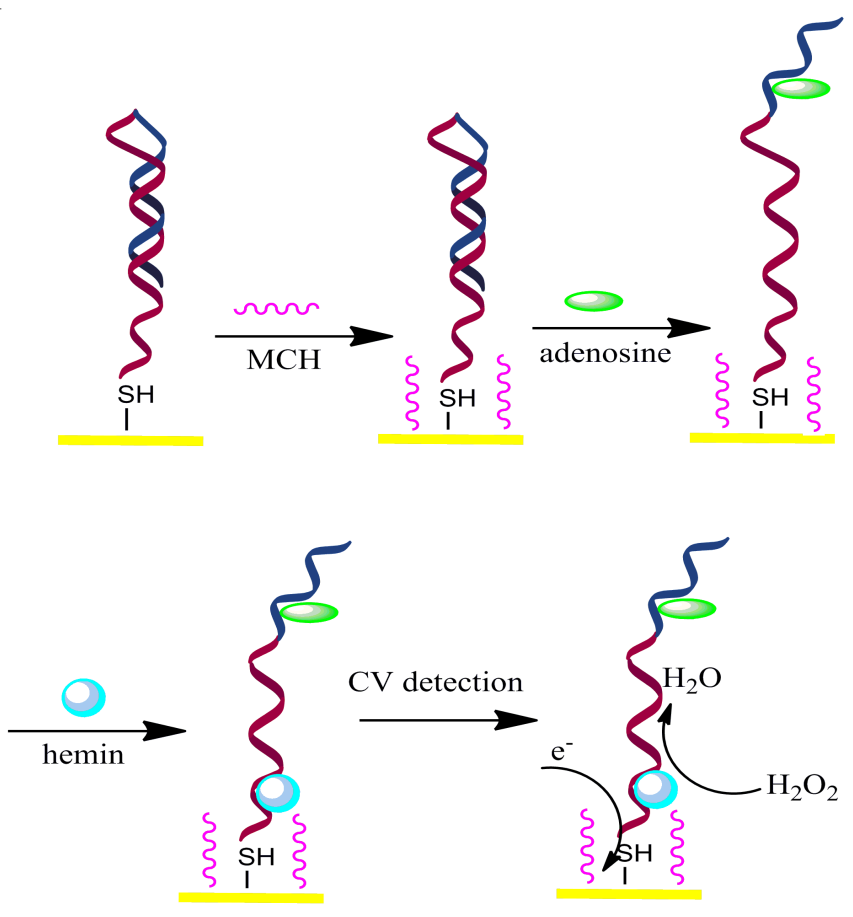

Scheme-I:Proposed scheme for the illustration of the label-free aptasensor for detection of adenosine 
and higher cathodic current is expected. On the basis of this characteristic, our assay can provide quantitative measurements for adenosine concentration.

Optimization of the experimental condition: There are various factors could influence the effect for the detection of adenosine by the G-quadruplex-hemin DNAzyme based aptasensor designed by us, such as the density of the hairpin aptamer immobilized on the gold electrode, incubation time and concentrations of hemin and $\mathrm{H}_{2} \mathrm{O}_{2}$. Thus, it is necessary to optimize these relevant experimental variables.

Optimization of the harpin aptamer density immobilized on electrode: Considering that the density of the hairpin aptamer on the gold electrode surface may affect the sensitivity and performance of the aptasensor, we carried out an experimental optimization for the hairpin aptamer surface density on the gold electrode. In the process, we fixed the concentration of adenosine at $10 \mu \mathrm{M}$ and varied the concentration of hairpin aptamer for immobilization. The current responses for different aptamer concentration and the same analyte concentration are shown in Fig. 1. As can be seen, the aptasensor with the aptamer concentration of $3 \mu \mathrm{M}$ shows the highest current response. This is probably due to the stronger steric hindrance effect of the hairpin aptamer at higher immobilization concentration $(>3 \mu \mathrm{M})$. On the contrary, low concentration of hairpin aptamer can provide more space for unfolding of the hairpin probe. Based on Fig. 1, an optimal hairpin aptamer concentration of $3 \mathrm{mM}$ was chosen for immobilization.

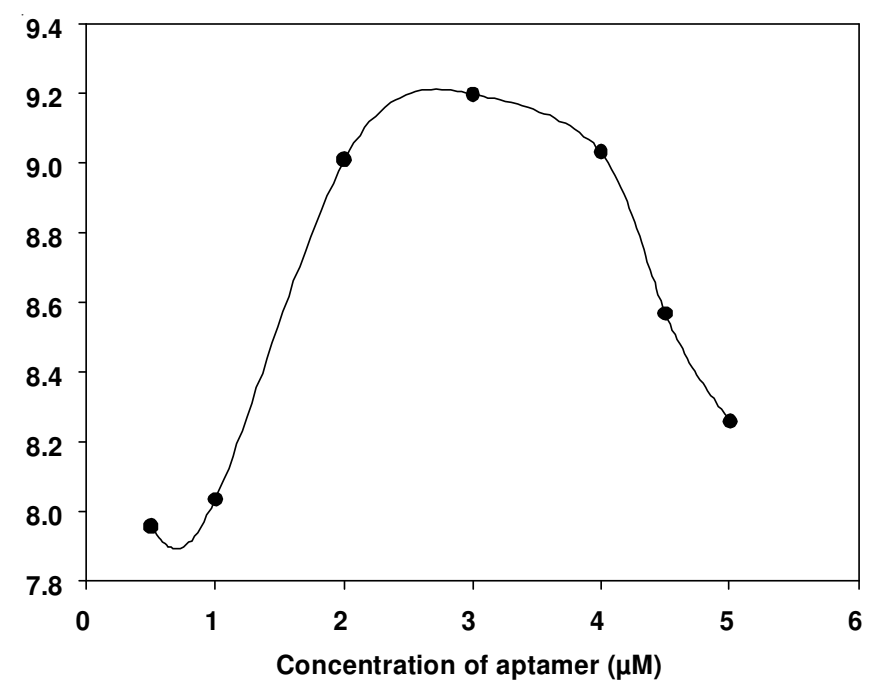

Fig. 1. Effect of the hairpin aptamer surface density upon the current response for adenosine detection. The measurements were performed in a $10 \mathrm{mM}$ HEPES buffer solution $(100 \mathrm{mM} \mathrm{NaCl}, 50$ $\mathrm{mM} \mathrm{KCl}, \mathrm{pH}$ 7.2) that included hemin, $1 \mu \mathrm{M}, \mathrm{H}_{2} \mathrm{O}_{2}, 1 \mathrm{mM}$ and adenosine, $10 \mu \mathrm{M}$

Effect of the incubation time: The effect of incubation time (the reaction time between the hairpin aptamer and adenosine) on the performance of the sensor was also investigated. Fig. 2 shows the time-dependent voltammetric responses of the hairpin aptamer-functionalized gold electrode upon its interaction with adenosine, $10 \mu \mathrm{M}$. As the time of interaction is prolonged, the electrocatalytic cathodic currents are intensified, consistent with the formation of higher degrees of opened hairpin. the current response ascends with the increase of the incubation time before $60 \mathrm{~min}$ and then to be stable, shows that all the hairpin structure were opened. Therefore, a incubation interval of 60 min was selected in the subsequent study.

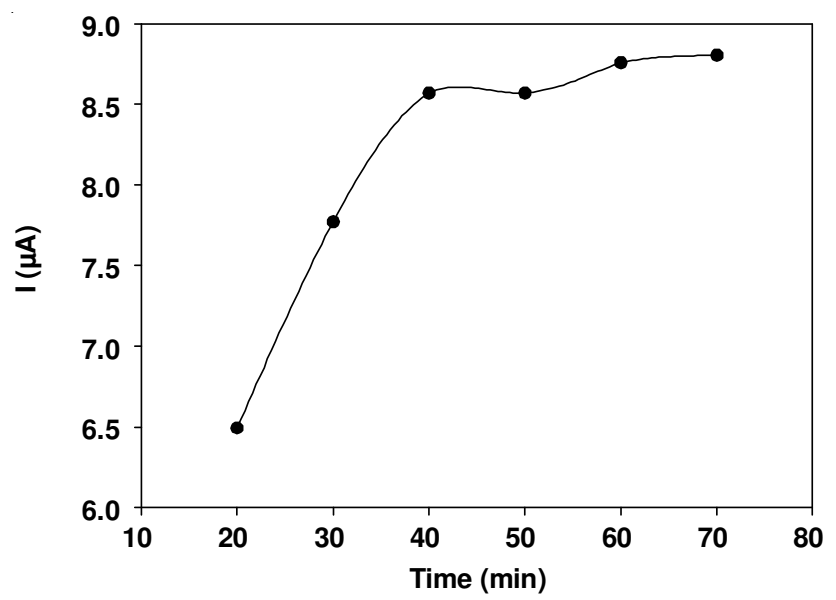

Fig. 2. Current responses of the electrocatalytic reduction of $\mathrm{H}_{2} \mathrm{O}_{2}$ by a HRP-mimicking DNAzyme caged in the DNA hairpin structure linked to the Au electrode and activated in the presence of a 1060 $\mu \mathrm{M}$ adenosine. Prior to the measurements, the electrode was allowed to react with adenosine for $20,30,40,50,60$ and $70 \mathrm{~min}$

Optimization of hemin concentration: We have also examined the effect of hemin concentration on the bioelectrocatalytic reduction of $\mathrm{H}_{2} \mathrm{O}_{2}$. We find that as the concentration of hemin increases, the bioelectrocatalytic currents corresponding to the reduction of $\mathrm{H}_{2} \mathrm{O}_{2}$ are enhanced and they level off to a saturation value at a hemin concentration corresponding to $2 \mu \mathrm{M}$. Accordingly, in all subsequent experiments, we used this concentration of hemin.

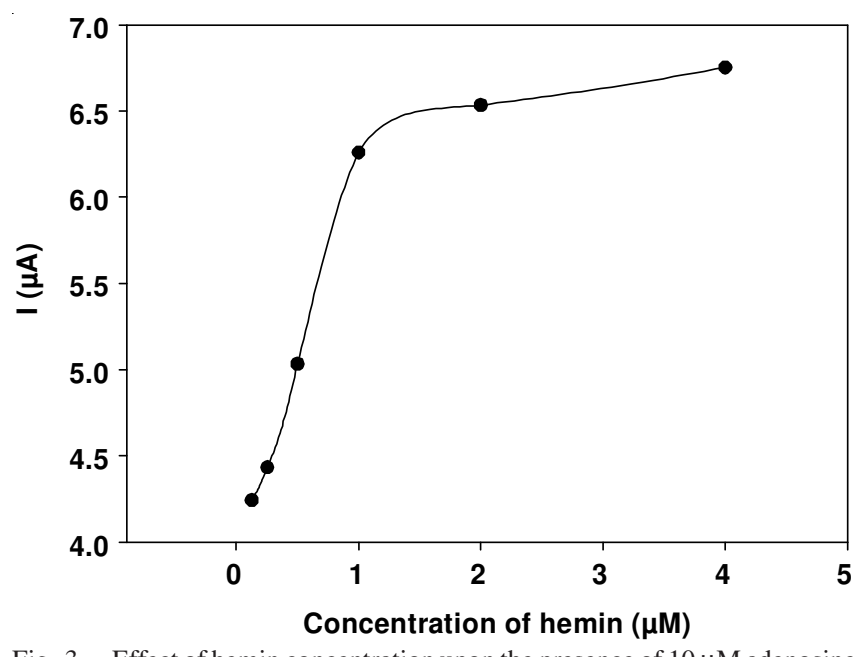

Fig. 3. Effect of hemin concentration upon the presence of $10 \mu \mathrm{M}$ adenosine

Analytical performance: The sensitivity of our assay protocol based on peroxidase mimicking DNAzyme amplification was investigated under the optimum experimental conditions by varying the target concentration. As displayed in Fig. 4(A), as the concentration of adenosine increases from 0 to $30 \mu \mathrm{M}$, the electro catalytic cathodic currents generated by the sensing electrode are intensified, consistent with a higher degree of the opening of the hairpin structure. When the 
adenosine concentration increased to $30 \mu \mathrm{M}$ or higher, the cathodic current signal starts to level off, suggesting the saturation of the sensing surface. The corresponding calibration curve for the adenosine over the $0 \mu \mathrm{M}$ to $50 \mu \mathrm{M}$ range is shown in Fig. 4(B). The detection limit can be estimated to be $0.25 \mu \mathrm{M}$ according to the characteristic current responses of the sensor for the presence of $0.25 \mu \mathrm{M}$ and zero adenosine, respectively.
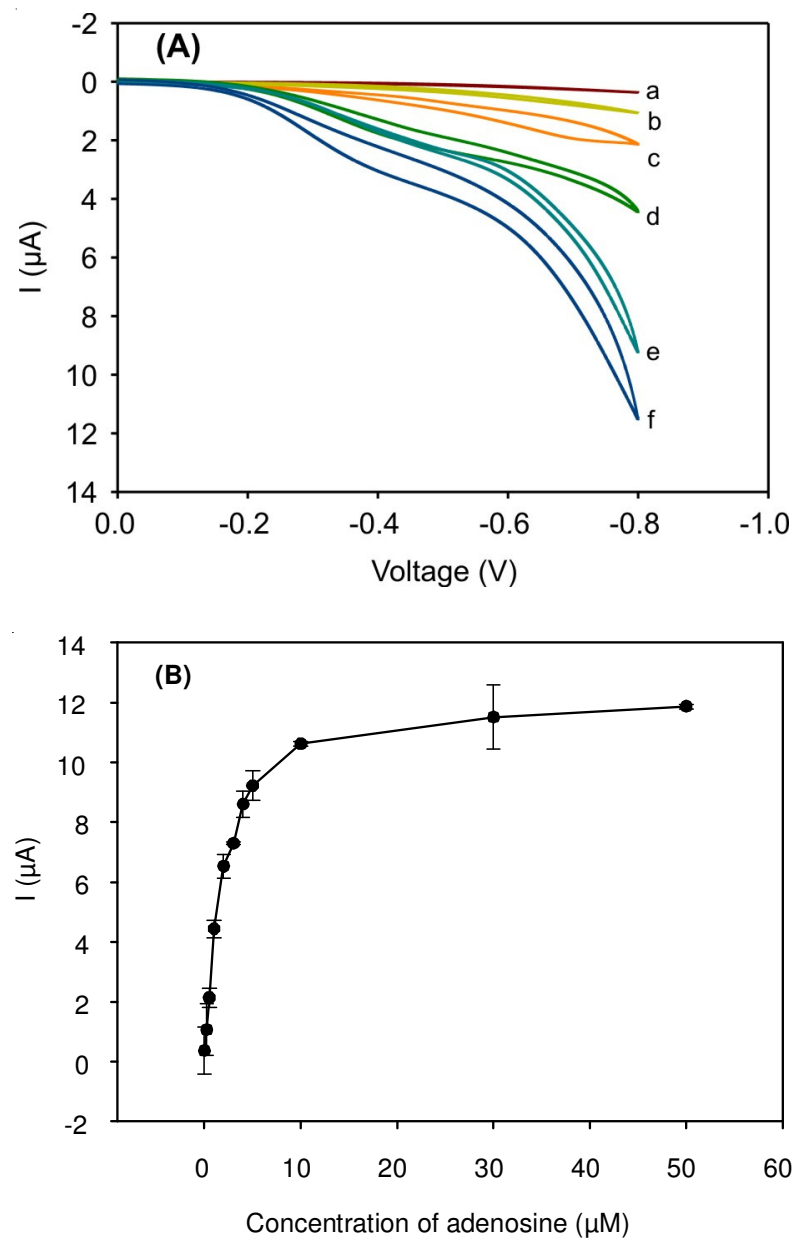

Fig. 4. (A) Electrocatalytic reduction of $\mathrm{H}_{2} \mathrm{O}_{2}$ by the adenosine-activated HRP-mimicking DNAzyme caged in the DNA hairpin structure linked to a Au electrode, in the presence of variable concentrations of adenosine: (a) 0, (b) 0.25, (c) 0.50, (d) 1 , (e) $30 \mu \mathrm{M}$. (B) The calibration curve corresponding to the electro catalytic currents, at $\mathrm{E}=-0.8 \mathrm{~V} v s$. SCE, at the different concentrations of adenosine. The measurements were performed in a HEPES buffer solution 10 mM HEPES buffer solution ( $100 \mathrm{mM} \mathrm{NaCl}, 50 \mathrm{mM} \mathrm{KCl}, \mathrm{pH} 7.2)$ that included hemin, $1 \mu \mathrm{M}$ and $\mathrm{H}_{2} \mathrm{O}_{2}, 1 \mathrm{mM}$. (The analytical performances of the sensor were investigated under the optimum experimental conditions, error bars were deduced from $\mathrm{N}=3$ experiments)

Interference study: In order to evaluate the selectivity of the proposed sensor for the detection of adenosine, comparative trials were carried out by using uridine $(10 \mathrm{mM})$, guanosine $(10 \mathrm{mM})$, cytidine $(10 \mathrm{mM})$ as the potential interference molecules to test the selectivity of the proposed aptasensor. As shown in Fig. 5, it is observed that the presence of $10 \mu \mathrm{M}$ target leads to a dramatic cathodic current change. However, when the target adenosine is replaced by uridine, guanosine, cytidine at even 1000 fold high concentrations, the change of cathodic current is insignificant when compared with the blank test (no adenosine in the testing buffer). These results suggest that our aptasensor based on the peroxidasemimicking DNAzyme is highly specific to adenosine, i.e. high selectivity can be observed.

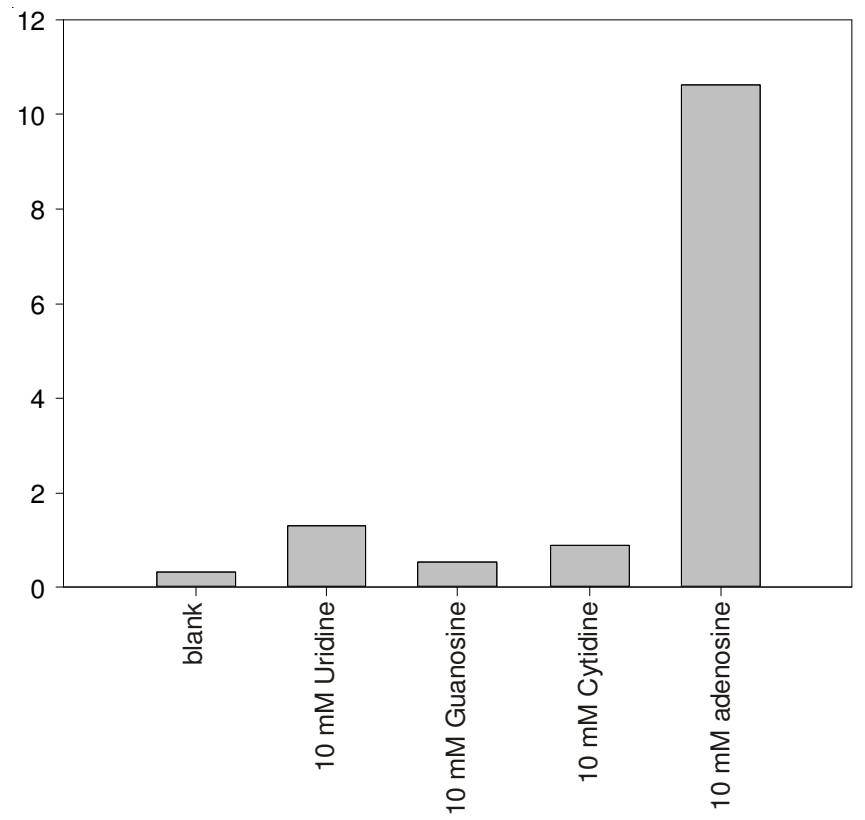

Fig. 5. Differences in the current responses of the sensor with the addition of different nucleosides. The results show that only adenosine caused a marked response and other nucleoside has no obvious interference, suggesting that this proposed sensing system is specific for the detection of adenosine

\section{Conclusion}

In summary, a novel label-free fluorescent aptasensor for the determination of adenosine was developed, using the integration of the DNAzyme sequence into the aptamer hairpin probe and subsequent formation of the hemin/G-quadruplex peroxidase-mimicking DNAzyme, leading to a direct catalytic amplification of the analytical signal in a label-free fashion with the fast development of the aptamer technology. The proposed strategy is expected to hold great potential for sensitive and selective detection of adenosine.

\section{ACKNOWLEDGEMENTS}

This work was supported by the Nature Science Fund of Naval University of Engineering (No. HGDQNJJ13158). The authors also acknowledged the corperation from the teachers (Ru-Qin Yu, Guo-Li Shen, Hai-Long Wu, Jian-Hui Jiang) at the State Key Laboratory of Chemo/Biosensing and Chemometrics at Hunan University.

\section{REFERENCES}

1. R. Gill, M. Zayats and I. Willner, Angew. Chem., 120, 7714 (2008).

2. J. Wang, G. Liu and A. Merkoçi, J. Am. Chem. Soc., 125, 3214 (2003).

3. E. Sharon, R. Freeman, R. Tel-Vered and I. Willner, Electroanalysis, 21,1291 (2009)

4. F. Patolsky, K.T. Ranjit, A. Lichtenstein and I. Willner, Chem. Commun., 1025 (2000).

5. D.J. Caruana and A. Heller, J. Am. Chem. Soc., 121, 769 (1999).

6. R. Polsky, R. Gill, L. Kaganovsky and I. Willner, Anal. Chem., 78, 2268 (2006). 
7. G. Pelossof, R. Tel-Vered, X. Q. Liu and I. Willner, Chem. Eur. J., 17, 8904 (2011).

8. E. Sharon, R. Freeman and I. Willner, Anal. chem., 82, 7073 (2010).

9. S. Shimron, F. Wang, R. Orbach and I. Willner, Anal. Chem., 84, 1042 (2011)

10. H. Needleman, Annu. Rev. Med., 55, 209 (2004).

11. Y. Liu, Z. Liu, Y. Wang, J. Dai, J. Gao, J. Xie and Y. Yan, Microchim. Acta, 172, 309 (2011).

12. X. H. Zhao, R.M. Kong, X.B. Zhang, H.M. Meng, W.N. Liu, W. Tan, G.L. Shen and R.Q. Yu, Anal. Chem., 83, 5062 (2011).

13. R.R. Breaker and G.F. Joyce, Chem. Biol., 1, 223 (1994).

14. T. J. Yim, J. Liu, Y. Lu, R. S. Kane and J. S. Dordick, J. Am. Chem. Soc., 127,12200 (2005)

15. X.B. Yin, TrAC Trends Anal. Chem., 33, 81 (2012).

16. H. Zhang, B. Jiang, Y. Xiang, Y. Chai and R. Yuan, Analyst, 137, 1020 (2012).
17. X. Fan, H. Li, J. Zhao, F. Lin, L. Zhang, Y. Zhang and S. Yao, Talanta, 89, 57 (2011).

18. D. Li, B. Shlyahovsky, J. Elbaz and I. Willner, J. Am. Chem. Soc., 129, 5804 (2007)

19. G. Pelossof, R. Tel-Vered, J. Elbaz and I. Willner, Anal. Chem., 82, 4396 (2010)

20. I. Willner, B. Shlyahovsky, M. Zayats and B. Willner, Chem. Soc. Rev., 37,1153 (2008)

21. Y. Xiao, V. Pavlov, T. Niazov, A. Dishon, M. Kotler and I. Willner, J. Am. Chem. Soc., 126, 7430 (2004).

22. E. Katz, B. Willner and I. Willner, in eds.: F.S. Emil Palecek and J. Wang, Amplified Electrochemical and Photoelectrochemical Analysis of DNA, In: Perspectives in Bioanalysis, Elsevier, Vol. 1, pp. 195-246 (2005). 